\title{
Ruth Stawarz-Luginbühl, Un théâtre de l'épreuve. Tragédies huguenotes en marge des guerres de religion en France (1550-1573)
}

\section{Michele Mastroianni}

\section{(2) OpenEdition \\ Journals}

\section{Edizione digitale}

URL: http://journals.openedition.org/studifrancesi/2115

DOI: $10.4000 /$ studifrancesi.2115

ISSN: 2421-5856

\section{Editore}

Rosenberg \& Sellier

\section{Edizione cartacea}

Data di pubblicazione: 1 aprile 2014

Paginazione: 132-133

ISSN: 0039-2944

\section{Notizia bibliografica digitale}

Michele Mastroianni, « Ruth Stawarz-Luginbühl, Un théâtre de l'épreuve. Tragédies huguenotes en marge des guerres de religion en France (1550-1573) », Studi Francesi [Online], 172 (LVIII | I) | 2014, online dal 01 avril 2014, consultato il 18 septembre 2020. URL : http://journals.openedition.org/studifrancesi/2115 DOI : https://doi.org/10.4000/studifrancesi.2115

\section{Questo documento è stato generato automaticamente il 18 settembre 2020.}

\section{cc) $(9)$}

Studi Francesi è distribuita con Licenza Creative Commons Attribuzione - Non commerciale - Non opere derivate 4.0 Internazionale. 


\title{
Ruth Stawarz-Luginbühl, Un théâtre de l'épreuve. Tragédies huguenotes en marge des guerres de religion en France (1550-1573)
}

\author{
Michele Mastroianni
}

\section{NOTIZIA}

RUTH STAWARZ-LUGINBÜHL, Un théâtre de l'épreuve. Tragédies huguenotes en marge des guerres de religion en France (1550-1573), Genève, Droz, 2012 («Travaux d'Humanisme et Renaissance», n. DV), pp. 696.

Come già aveva evidenziato Raymond Lebègue, con la sua grande thèse del 1929, il periodo che val dal $1550 \mathrm{al} 1573$ è caratterizzato, in Francia, dalla fioritura di un teatro che prendendo la relève dei mystères vuole costruire la pièce teatrale religiosa secondo modelli e schemi della tragedia classica, che, come genere, è al centro della rinascita delle forme letterarie antiche. Di questo teatro sacro abbiamo oggi pubblicazioni critiche moderne: non soltanto edizioni sparse, ma anche edizioni raccolte in un grande corpus del teatro francese cinquecentesco (giunto oggi al ventesimo volume) e precisamente quel Théâtre Français de la Renaissance fondato, nel 1986, da Enea Balmas e Michel Dassonville e diretto oggi da Rosanna Gorris, coadiuvata da un'équipe di specialisti del teatro cinquecentesco (in particolare della tragédie sainte) quali Nerina Clerici Balmas, Anna Bettoni, Mariangela Miotti. Questi strumenti facilitano raffronti fra le due filières, quella del teatro sacro e quella del teatro profano. Negli ultimi decenni, poi, si è intensificato il discorso sulla tragédie sainte e si è centrata l'attenzione su quel dibattito che nel secondo Cinquecento si era sviluppato sulla scorta della riflessione aristotelica circa l'essenza del genere tragico: il dibattito sulla possibilità di creare una tragedia corrispondente a quella dei classici servendosi di storie bibliche e ispirandosi a una teologia cristiana della storia. Si tratta di una problematica affrontata 
da Enea Balmas e dalla sua scuola milanese, da Olivier Millet, particolarmente attento ai rapporti tra teologia e letteratura nel Cinquecento, da Charles Mazouer, il grande ordinatore e sistematore della storia del teatro francese rinascimentale, barocco e classico.

2 La silloge di testi tragici oggetto dell'analisi di R. Stawarz-Luginbühl - tutti appartenenti all'area della Riforma - è la seguente: Théodore de Bèze, Abraham sacrifiant (1550); Joachim de Coignac, La Desconfiture de Goliath (1551); A. de La Croix (Antoine de La Roche-Chandieu), Tragi-comedie (1561); André de Rivaudeau, Aman (1559-1561/1566); Louis Des Masures, Tragedies sainctes: David combattant - David triomphant - David fugitif (1562-1566); Jean de La Taille, Saül le Furieux (1562-1572) - La Famine, ou les Gabeonites (1573). Della pièce di J. de Coignac e di quella di A. de La Croix non esiste edizione moderna (sono comunque entrambe consultabili su BnF-Gallica). Sulla base di queste pièces l'A. riprende il discorso sulla riflessione rinascimentale relativa al tragico, evidenziando come il fatto che il genere della tragédie sainte appartenga, all'origine, all'area culturale protestante sia strettamente connesso a una lettura 'tragica' della storia della propria comunità: comunità sulla quale si proietta una nozione squisitamente teologica, quella della prova cui è sottoposto l'eletto, ovvero il tema della 'caduta'. Il che comporta una situazione di crisi che viene interpretata come il corrispondente della 'crisi' drammatica su cui si costruisce la tragedia classica. Come viene indicato nell'introduzione, «l'idée qui est à la base de ce travail est d'examiner de près ce moment dramatique trop rarement scruté par la critique qu'est le passage effectif ou manqué - du désespoir à la confiance, cette crise, de nature intellectuelle, existentielle et spirituelle, qui place le protagoniste et la communauté à laquelle il appartient, solidairement porteurs d'une promesse divine, face à la rupture apparente du contrat initial, face à la béance angoissante qui les sépare d'une hypothétique délivrance» (p. 17). Infatti, tenendo presente e discutendo la letteratura critica precedente, l'A. lavora intorno a questo soggetto: «la question de savoir par quel moyen dramaturgique la souffrance, sous la forme rhétorique de la plainte, pourra être modulée et finalement endiguée dans son déploiement temporel et logique pour être redirigée vers le pôle opposé aux affects négatifs, à savoir vers la joie et la confiance; ou, autrement dit, comment il est possible de représenter un cycle d'épreuve complet, de la 'chute' au désespoir et du désespoir à la confiance sans faire intervenir Dieu au moment du revirement - mais tout en faisant sentir sa présence en coulisse» (pp. 35-36). Sul piano metodologico, si insiste sulla necessità di accordare un'assoluta prevalenza al testo, premunendosi «contre une lecture qui dénierait d'avance aux tragédies bibliques toute forme d'ouverture sémantique sous prétexte que les récits dont elles s'inspirent seraient eux-mêmes, par essence, dépourvus de polysémie parce qu'ils racontent l'histoire du salut, parce que leur signification religieuse, considérée comme close, doit nécessairement prendre le pas sur les potentialités sémantiques plus ouvertes qu'une analyse de type littéraire est capable de mettre au jour» (p. 41). Si tratterebbe peraltro di una tentazione particolarmente forte trattandosi di autori appartenenti al calvinismo: infatti, la dottrina della predestinazione potrebbe essere facilmente intesa come l'equivalente della fatalità tragica. Tuttavia, per Ruth StawarzLuginbühl, l'autore umanista fa consistere il tragico più che sulla lotta, persa fin dall'inizio, di un eroe contro il potere ineluttabile del fato, sulla precarietà costitutiva di qualsiasi creatura umana, in un ottica morale, politica e religiosa. Per cui «dans une perspective herméneutique affirmer que l'on sait d'avance que la colère de Dieu finira par écraser celui qu'il a reprouvé de toute éternité ou, au contraire, se dire persuadé 
d'avance que Dieu n'abandonnera jamais les siens revient dans tout les cas à aplatir le texte, à raboter ses aspérités sémantiques en lui imposant, de façon unilatérale, la causalité rétrospective du lecteur» (p. 43). Verrà quindi adottato, nell'analisi, il punto di vista dei personaggi, ignari dell'esito finale e impegnati nella lotta morale. Come l'A. ancora sottolinea, quale direttiva di metodo, «ce n'est qu'à condition d'adopter cette posture herméneutique qu'on sera à même de penser la discordance de l'intérieur, c'està-dire de comprendre de quelle manière se construit la réponse éthique et spirituelle du protagoniste à l'épreuve qu'il traverse sans savoir où il va, de quelle manière donc se trame la présence absente de Dieu dans le texte dramatique, privé par définition des ressources plus souples et plus nombreuses dont peut se prévaloir la narration» (p. 45).

Per quanto riguarda l'impianto del volume, dopo un'introduzione che, ricostruito l'état présent des études, confronta le idee di fondo sottostanti all'indagine con le grandi linee degli studî moderni sulla tragedia del Rinascimento, si ha un primo capitolo (Problématique et enjeux de la tentation: une herméneutique du renversement, pp. 51-142) che tenta di formulare una sintesi dei problemi ideologici e drammaturgici delle nozioni di tentation ed épreuve. In effetti, dall'analisi dell'azione nel suo svolgersi e della caratterizzazione del personaggio, l'A. conclude che gli interrogativi posti dalla ricerca dell'essenza del tragico «reçoivent toujours dans le cadre d'une vision théocentrique et providentielle du monde, une réponse presque invariable: la tentation ou l'épreuve. L'homme est affligé non pas pour son mal mais pour son bien; autrement dit, le mal est pour lui l'occasion de montrer ce qu'il y a de bien en lui, de prouver aux dieux, qui son responsables de sa situation et qui le regardent, sa capacité à surmonter d'une manière ou d'une autre ce qui est susceptible de le détruire. Présente dans toute la littérature judéo-chrétienne, développée par certains philosophes antiques et notamment stoïciens, cette grille de lecture continue d'imprégner profondément les réflexions que les auteurs réformés consacrent à la problématique du mal» (p. 51). In una prima sezione vengono pertanto illustrate le definizioni e le tipologie della 'tentazione' dall'antichità al Rinascimento, ripercorrendo l'Antico e il Nuovo Testamento, i Padri e il De providentia senechiano. In una seconda sezione si evidenziano le modalità con cui queste tipologie diventano chiave ermeneutica delle vicende della Chiesa riformata- le tentations e le épreuves, appunto, del nuovo Israele. In una terza sezione si cerca di abbozzare un'articolazione possibile fra la tematica della tentazione e i criteri generici della tragedia. Sarà quello della tentazione dunque - per usare l'espressione di R. Stawarz-Luginbühl - il filo di Arianna del percorso che rintraccia le problematiche filosofiche, religiose, ermeneutiche e drammaturgiche nelle nove piéces studiate in altrettanti capitoli che sono vere e proprie monografie.

4 Siamo dunque in presenza di un lavoro che, al di là dell'originalità interpretativa, offre uno strumento di lavoro e di consultazione utilissimo a chi si occupa di tragédie sainte rinascimentale (e di tragedia in genere), in particolare perché riassume le principali filières critiche sull'argomento e aiuta la comprensione intertestuale delle tragedie analizzate. Ricca ed esauriente la bibliografia. 